\title{
A lealdade dos espectadores de futebol e seus antecedentes: uma revisão sistemática ${ }^{1}$
}

\section{Football spectator's loyalty and yours pedictors: a systematic review}

\section{Lealtad y antecedentes de los espectadores de fútbol: una revisión sistemática}

(iD) Victor Henrique Rodrigues Silva

Universidade de Pernambuco, Recife, Pernambuco, Brasil victorhenrique47@hotmail.com

iD Marcos Antonio Barros Filho

Universidade de Pernambuco, Recife, Pernambuco, Brasil marcos.barrosf@hotmail.com

iD Yves de Holanda Batista de Miranda Universidade de Pernambuco, Recife, Pernambuco, Brasil miranda95y@gmail.com

(iD) José Pedro Sarmento Universidade de Pernambuco, Recife, Pernambuco, Brasil psarmento@fade.up.pt

iD? Carlos Augusto Mulatinho de Queiroz Pedroso Universidade de Pernambuco, Recife, Pernambuco, Brasil carlos.mulatinho@upe.br

Resumo: O objetivo deste estudo foi identificar os antecedentes da lealdade dos torcedores de clubes de futebol. Foi realizada uma revisão sistemática utilizando artigos originais de 2009 a 2019, indexados na "Academic Search Ultimate", "Business Source Ultimate", "Scopus" e "Web of

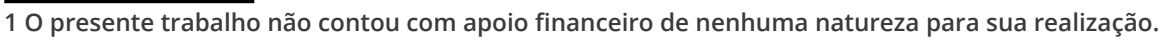


Science"; 166 foram encontrados e 21 incluídos. Nos resultados, a maioria dos artigos foi com base no contexto europeu. Em relação ao instrumento e análise, todos os estudos utilizaram questionário com escala Likert que variava entre 5 e 11 pontos. Análise de Equações Estruturais foi o principal método de análise dos dados. Como resultado, os principais preditores encontrados foram a satisfação e a identificação. Sugerese análises no contexto das Américas, em especial no Brasil.

Palavras-chave: Lealdade. Antecedentes. Espectador. Futebol.

Abstract: This study aimed to identify the antecedents of soccer club fans' loyalty. A systematic review was carried out, using original articles from 2009 to 2019, indexed in "Academic Search Ultimate", "Business Source Ultimate", "Scopus" and "Web of Science", 166 were found and 21 were included. In the results, most of the articles were in the European context. Regarding the instrument and analysis, all studies used a questionnaire with a Likert scale that varied between 5 and 11 points. Structural Equation Modeling was the main method of data analysis. As a result, the main predictors found were satisfaction and identification. Analyzes are suggested in the context of the Americas, especially in Brazil. Keywords: Loyalty; Antecedents; Spectators; Football.

Resumen: El objetivo fue identificar los antecedentes de la lealtad de los torcedores de clubes de fútbol. Fue realizóada una revisión sistemática, utilizando artículos originales de 2009 a 2019, indexados en "Academic Search Ultimate", "Business Source Ultimate", "Scopus" y "Web of Science", se encontraron 166 y se incluyeron 21 . En los resultados, la mayoría de los artículos estaban en el contexto europeo. En cuanto al instrumento y análisis, todos los estudios utilizaron un cuestionario con escala Likert que varió entre 5 y 11 puntos. El Modelo de Ecuaciones Estructurales fue el principal método de análisis de datos. Los principales predictores encontrados fueron la satisfacción y la identificación. Se sugieren análisis en el contexto de las Américas, especialmente en Brasil.

Palabras-clave: Lealtad; Antecedente; Espectadores; Fútbol

Submetido em: 14-08-2020

Aceito em: 20-05-2021 
A lealdade dos espectadores de futebol e seus antecedentes: uma revisão sistemática Victor Henrique Rodrigues Silva $•$ Marcos Antonio Barros Filho - Yves de Holanda Batista de Miranda • José Pedro Sarmento · Carlos Augusto Mulatinho de Queiroz Pedroso

\section{Introdução}

O aumento da procura dos espectadores por eventos esportivos no seu tempo livre faz com que os gestores ligados à indústria esportiva, principalmente ao futebol, o esporte mais consumido pela população mundial (por sua prática ou pelo entretenimento), se qualifiquem e aprimorem suas táticas para manter o espectador satisfeito e, consequentemente, com intenções comportamentais favoráveis (YOSHIDA; JAMES, 2010; BISCAIA; CORREIA; ROSADO; MENEZES, 2014).

Os clubes esportivos concorrem com outras opções de lazer para além do esporte (BAUER et al., 2005). Porém, o indivíduo que acompanha um clube esportivo traz consigo fatores para além do desempenho da equipe em si. Traz também a sensação de pertencimento e envolvimento com as comunidades de torcedores, favorecendo atitudes positivas em relação às intenções de recompra (FUNK; PASTORE, 2000; FUNK; JAMES; 2004).

Além disso, o desenvolvimento tecnológico e a facilidade de acesso a conteúdos dos clubes das mais diversas ligas, afastadas do ponto de vista geográfico, aumentaram, de forma exponencial, a concorrência entre essas organizações esportivas. Portanto, reitera-se a importância das ações de marketing realizadas pelos clubes para manter o torcedor compromissado com o seu clube (MADERER; HOLTBRÜGGE, 2018).

Diante desse cenário, estudos voltados para a o entendimento do comportamento do torcedor em relação aos serviços prestados pelas organizações começaram a surgir para acompanhar a rápida evolução da indústria do esporte (CHELLADURAl; CHANG, 2000; YOSHIDA; JAMES, 2011; BISCAIA et al., 2014; THEYSOHNHINZ; NOSWORTHY; KIRCHNER, 2009).

As intenções e o comportamento de consumo do torcedor podem manifestar o seu nível de lealdade e sofrer influência de diversos antecedentes, como: os serviços prestados pelas organizações, emoções, identificação com a equipe, o valor da marca, entre 
A lealdade dos espectadores de futebol e seus antecedentes: uma revisão sistemática Victor Henrique Rodrigues Silva - Marcos Antonio Barros Filho • Yves de Holanda Batista de Miranda • José Pedro Sarmento · Carlos Augusto Mulatinho de Queiroz Pedroso

outras (BISCAIA, 2016). Entende-se que os antecedentes são variáveis apontadas como preditoras diretas ou indiretas de uma consequência, nesse caso a lealdade (BISCAIA et al., 2014). Portanto, entender a importância dos antecedentes que influenciam no comportamento futuro são essenciais para as organizações, pois podem ajudar na tomada de decisão nas ações que visam a tornar o torcedor leal (MITTAL; KUMAR; TSIROS, 1999; BISCAIA et al., 2014).

Atualmente, há duas abordagens para mensurar a lealdade do torcedor: a lealdade atitudinal e a lealdade comportamental. A primeira, mais usada na literatura, busca compreender a lealdade do torcedor por meio das intenções de voltar a consumir os serviços e produtos do clube a partir de experiências passadas (KAYNAK; SALMAN; TATOGLU, 2008) e a segunda é voltada para a materialização do consumo (se consumiu e quantas vezes consumiu) (DICK; BASU, 1994; ZINS, 2001).

De acordo com Maderer e Holtbrügge (2018), diversos autores utilizam-se apenas da lealdade atitudinal para mensurar a lealdade dos consumidores. Porém, a lealdade comportamental é tão importante quanto a atitudinal, pois o indivíduo pode ter a intenção de ir aos jogos e consumir os serviços do clube, mas alguns constrangimentos (e. g., distância do estádio, preço dos produtos, renda mensal etc.) fazem o torcedor consumir com maior ou menor frequência (BISCAIA et al., 2014). Portanto, ter bons níveis de intenções não caracteriza que aquele determinado indivíduo irá consumir com frequência.

Diante de todo o cenário acima, este estudo teve como objetivo identificar quais os antecedentes da lealdade dos torcedores de clubes de futebol à luz da literatura, pois isso poderá fornecer elementos relevantes para os gestores trabalharem a relação dos torcedores com seus clubes e transformar tal relacionamento em atitudes e comportamentos positivos. 
A lealdade dos espectadores de futebol e seus antecedentes: uma revisão sistemática Victor Henrique Rodrigues Silva $•$ Marcos Antonio Barros Filho - Yves de Holanda Batista de Miranda • José Pedro Sarmento · Carlos Augusto Mulatinho de Queiroz Pedroso

\section{Métodos}

Trata-se de uma revisão sistemática, que se caracteriza pelo emprego de uma metodologia rigorosa em relação a uma revisão de literatura tradicional, em que o estabelecimento de etapas bem definidas no processo de recolha dos artigos e suas informações permitem a replicação do estudo (GOMES; CAMINHA, 2013; BATISTA; CUNHA, 2013).

A busca por artigos originais foi realizada em quatro bases de dados eletrônicas (Academic Search Ultimate, Business Source Ultimate, Scopus e Web of Science), utilizando-se a busca avançada para incluir estudos publicados entre janeiro de 2009 e dezembro de 2019. Optou-se por dividir os termos de busca em quatro grupos, com o intuito de selecionar o maior e mais adequado número de artigos possíveis. Os grupos estão descritos abaixo, no Quadro 1.

Quadro 1- Grupos e descritores de pesquisa avançada

\begin{tabular}{|c|c|c|c|c|c|c|}
\hline Grupo 1 (OR) & \multirow{7}{*}{ AND } & Grupo 2 (OR) & \multirow{7}{*}{ AND } & Grupo 3 (OR) & \multirow{7}{*}{ AND } & Grupo 4 (OR) \\
\hline Antecedents & & "Behavioral Intentions" & & Fans & & Soccer \\
\hline Determining & & "Consumer Behavior" & & Customer & & Football \\
\hline "Service Quality" & & "Purchase Intentions" & & Spectator & & \\
\hline Emotions & & Loyalty & & Consumer & & \\
\hline Satisfaction & & & & Attendees & & \\
\hline "Brand Association" & & & & & & \\
\hline
\end{tabular}

Fonte: os autores.

Para selecionar os artigos elegíveis, quatro critérios de exclusão foram utilizados: 1) a falta de relação com o tema; 2 ) estudos repetidos; 3) estar fora do contexto do futebol; 4) artigos de revisão, eventos acadêmicos, periódicos e notícias.

Ao aplicar os descritores na base de dados e utilizar os critérios definidos, 166 artigos foram encontrados. Após a leitura do título e do resumo, 131 artigos foram excluídos. Ao analisar os 35 restantes a partir da leitura completa dos artigos, 15 estudos fo- 
A lealdade dos espectadores de futebol e seus antecedentes: uma revisão sistemática Victor Henrique Rodrigues Silva - Marcos Antonio Barros Filho • Yves de Holanda Batista de Miranda • José Pedro Sarmento · Carlos Augusto Mulatinho de Queiroz Pedroso

ram excluídos. Ao analisar as referências bibliográficas dos artigos selecionados, foi incluído mais um artigo. Portanto, para este estudo, foram selecionados 21 artigos originais. Para maior compreensão, um fluxograma das etapas da busca e o critério adotado para a exclusão dos artigos é apresentado abaixo, na Figura 1.

Figura 1 - Fluxograma

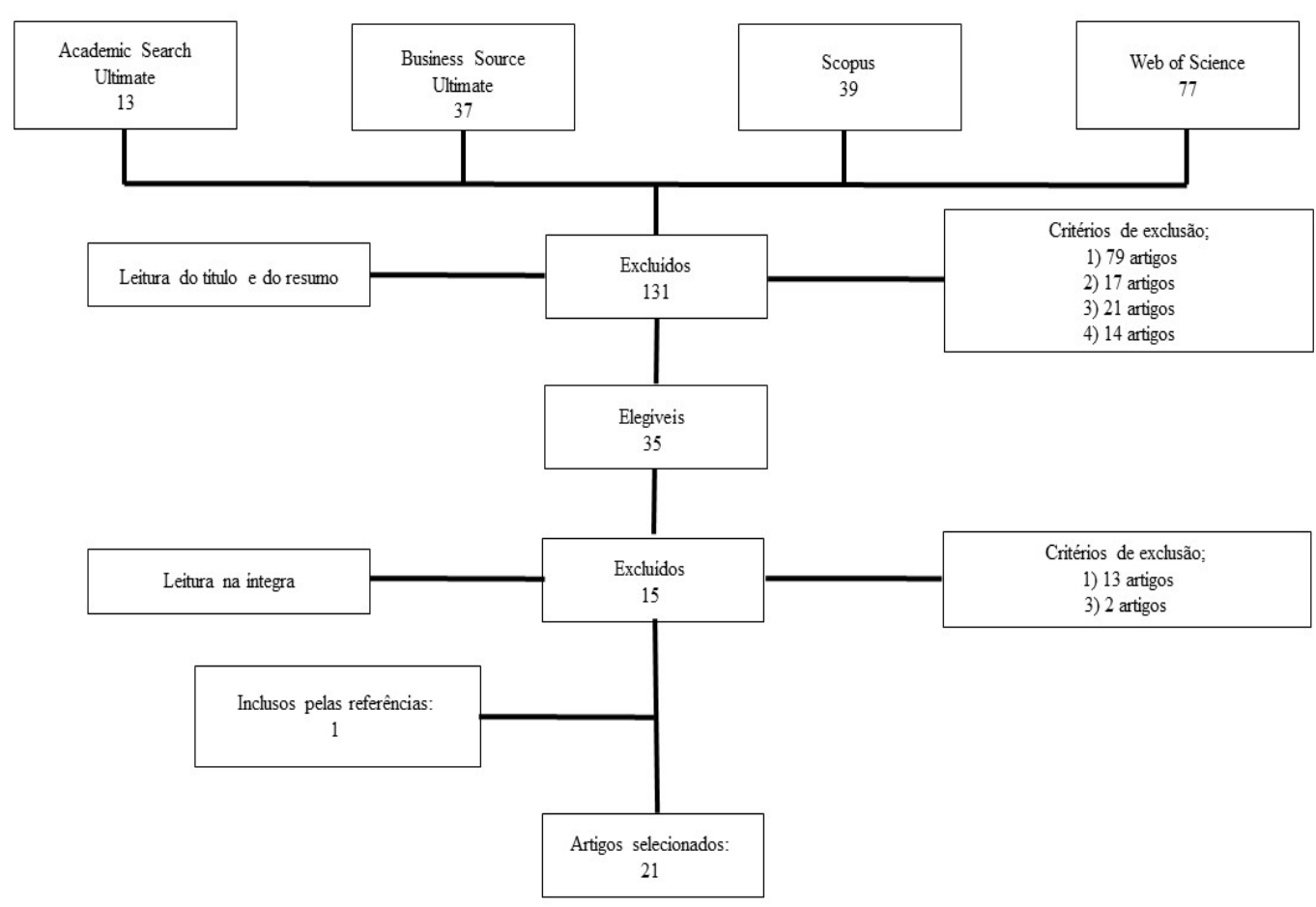

Fonte: os autores.

\section{Resultados}

A partir dos resultados da busca, observou-se que, nos últimos dez anos, dez estudos foram realizados no contexto europeu (Portugal, Polônia, Croácia, Grécia e Alemanha), seis no contexto asiático (Irã, Japão, Malásia e Tailândia), três estudos na América do Sul (Brasil) e um na Oceania (Austrália). Além disso, um estudo foi realizado em dez países distintos (Alemanha, Brasil, China 
A lealdade dos espectadores de futebol e seus antecedentes: uma revisão sistemática Victor Henrique Rodrigues Silva $•$ Marcos Antonio Barros Filho - Yves de Holanda Batista de Miranda • José Pedro Sarmento · Carlos Augusto Mulatinho de Queiroz Pedroso

Espanha, EUA, França, Índia, Inglaterra, Itália e Rússia). Não foram encontrados estudos exclusivos do contexto Africano e da América do Norte. Em ordem cronológica, o Quadro 2 apresenta os estudos encontrados e o país onde o estudo foi realizado.

\section{Quadro 2 - Estudos selecionados e país do contexto}

\begin{tabular}{|l|l|}
\hline \multicolumn{1}{|c|}{ Autores (ano) } & \multicolumn{1}{c|}{ País do contexto } \\
\hline Biscaia, Correia, Rosado, Maroco e Ross (2012) & Portugal \\
\hline Yoshida e Gordon (2012) & Japão \\
\hline Theodorakis, Wann e Weaver (2012) & Grécia \\
\hline Theodorakis, Alexandris, Tsigilis e Karvounis (2013) & Grécia \\
\hline Biscaia, Correia, Yoshida, Rosado e Marôco (2013) & Portugal \\
\hline Fernandes e Neves (2014) & Portugal \\
\hline $\begin{array}{l}\text { Biscaia, Ross, Yoshida, Correia, Rosado e Marôco } \\
\text { (2016) }\end{array}$ & Portugal \\
\hline Foroughi, Nikbin, Hyun e Iranmanesh (2015) & Irã \\
\hline Yoshida, Heere e Gordon (2015) & Japão \\
\hline Ebrahimi e Fadaei (2016) & Irã \\
\hline Oman, Pepur e Arneric (2016) & Croácia \\
\hline Rodrigues, Sousa e Fagundes (2017) & Brasil \\
\hline Kunkel, Funk e Lock (2017) & Austrália \\
\hline Marquetto, Pinto, Grohmann e Battistella (2017) & Brasil \\
\hline Sousa e Vieira (2018) & Portugal \\
\hline Silveira, Cardoso e Silva (2018) & Brasil \\
\hline Maderer e Holtbrügge (2018) & Alemanha, Brasil, China Espanha, EUA, \\
\hline Phonthanukitithaworn e Sellitto (2018) & Tailândia \\
\hline Kościołek (2019) & Polônia \\
\hline Woratschek, Horbel e Popp (2019) & Alemanha \\
\hline Foroughi, Ramayah e Iranmanesh (2019) & Malásia \\
\hline
\end{tabular}

Fonte: os autores.

Em relação à análise estatística, a maioria dos estudos (17) optou por utilizar a análise de equações estruturais (AEE), enquanto três estudos utilizaram a análise de regressão e um estudo utilizou a correlação de Spearman. Relativamente ao instrumento, todos os estudos utilizaram questionário de escala Likert, que variou 
A lealdade dos espectadores de futebol e seus antecedentes: uma revisão sistemática Victor Henrique Rodrigues Silva $•$ Marcos Antonio Barros Filho - Yves de Holanda Batista de Miranda • José Pedro Sarmento · Carlos Augusto Mulatinho de Queiroz Pedroso

entre 5, 7 e 11 pontos, sendo a de 7 pontos (16 estudos) a mais utilizada.

Além de apresentar informações sobre o tamanho da amostra, instrumento e principal tipo de análise, o Quadro 3 também apresenta quais foram os antecedentes que influenciaram na lealdade do torcedor, de acordo com a ordem cronológica dos estudos.

\section{Quadro 3 - Principais dados dos artigos selecionados para a revisão}

\begin{tabular}{|c|c|c|c|}
\hline Autores (ano) & $\begin{array}{l}\text { Instrumento e } \\
\text { amostra (n) }\end{array}$ & $\begin{array}{c}\text { Principal tipo de } \\
\text { análise }\end{array}$ & $\begin{array}{l}\text { Antecedentes } \\
\text { influentes }\end{array}$ \\
\hline $\begin{array}{l}\text { Biscaia, Correia, Rosado, } \\
\text { Maroco e Ross (2012). }\end{array}$ & $\begin{array}{l}\text { Questionário com } \\
\text { escala Likert de } 5 \\
\text { pontos ( } n=466) \text {. }\end{array}$ & $\begin{array}{l}\text { Análise de equa- } \\
\text { ções estruturais. }\end{array}$ & Alegria e satisfação. \\
\hline Yoshida e Gordon (2012). & $\begin{array}{l}\text { Questionário com } \\
\text { escala Likert de } 7 \\
\text { pontos (n=383). }\end{array}$ & $\begin{array}{l}\text { Análise de equa- } \\
\text { ções estruturais. }\end{array}$ & $\begin{array}{l}\text { Relacionamento e va- } \\
\text { lor da marca. }\end{array}$ \\
\hline $\begin{array}{l}\text { Theodorakis, Wann e Weaver } \\
\text { (2012). }\end{array}$ & $\begin{array}{l}\text { Questionário com } \\
\text { escala Likert de } 7 \\
\text { pontos (n=202). }\end{array}$ & $\begin{array}{l}\text { Análise de equa- } \\
\text { ções estruturais. }\end{array}$ & Identificação. \\
\hline $\begin{array}{l}\text { Theodorakis, Alexandris, } \\
\text { Tsigilis e Karvounis (2013). }\end{array}$ & $\begin{array}{l}\text { Questionário com } \\
\text { escala Likert de } 7 \\
\text { pontos ( } n=350) \text {. }\end{array}$ & $\begin{array}{l}\text { Análise de equa- } \\
\text { ções estruturais. }\end{array}$ & Satisfação. \\
\hline $\begin{array}{l}\text { Biscaia, Correia, Yoshida, } \\
\text { Rosado e Marôco (2013). }\end{array}$ & $\begin{array}{l}\text { Questionário com } \\
\text { escala Likert de } 7 \\
\text { pontos ( } n=1355) .\end{array}$ & $\begin{array}{l}\text { Análise de equa- } \\
\text { ções estruturais. }\end{array}$ & $\begin{array}{l}\text { Segurança, experiên- } \\
\text { cia com a multidão, } \\
\text { preço dos bilhetes e } \\
\text { satisfação. }\end{array}$ \\
\hline Fernandes e Neves (2014). & $\begin{array}{l}\text { Questionário com } \\
\text { escala Likert de } 7 \\
\text { pontos }(n=331) .\end{array}$ & $\begin{array}{l}\text { Regressão linear } \\
\text { e múltipla. }\end{array}$ & Satisfação. \\
\hline $\begin{array}{l}\text { Biscaia, Ross, Yoshida, Correia, } \\
\text { Rosado e Marôco (2016) }\end{array}$ & $\begin{array}{l}\text { Questionário com } \\
\text { escala Likert de } 7 \\
\text { pontos ( } n=2287)\end{array}$ & $\begin{array}{l}\text { Análise de equa- } \\
\text { ções estruturais. }\end{array}$ & $\begin{array}{l}\text { Comunidade de torce- } \\
\text { dores e internacionali- } \\
\text { zação da marca. }\end{array}$ \\
\hline $\begin{array}{l}\text { Foroughi, Nikbin, Hyun \& } \\
\text { Iranmanesh (2015). }\end{array}$ & $\begin{array}{l}\text { Questionário com } \\
\text { escala Likert de } 7 \\
\text { pontos (n=233). }\end{array}$ & $\begin{array}{l}\text { Análise de equa- } \\
\text { ções estruturais. }\end{array}$ & Emoções. \\
\hline $\begin{array}{l}\text { Yoshida, Heere e Gordon } \\
\text { (2015). }\end{array}$ & $\begin{array}{l}\text { Questionário com } \\
\text { escala Likert de } 7 \\
\text { pontos ( } n=233) .\end{array}$ & $\begin{array}{l}\text { Análise de equa- } \\
\text { ções estruturais. }\end{array}$ & $\begin{array}{l}\text { Comunidade de torce- } \\
\text { dores e identificação. }\end{array}$ \\
\hline Ebrahimi e Fadaei (2016). & $\begin{array}{l}\text { Questionário com } \\
\text { escala Likert de } 5 \\
\text { pontos ( } n=480) \text {. }\end{array}$ & $\begin{array}{l}\text { Análise de equa- } \\
\text { ções estruturais. }\end{array}$ & $\begin{array}{l}\text { Confiança e envolvi- } \\
\text { mento. }\end{array}$ \\
\hline
\end{tabular}


A lealdade dos espectadores de futebol e seus antecedentes: uma revisão sistemática Victor Henrique Rodrigues Silva $•$ Marcos Antonio Barros Filho - Yves de Holanda Batista de Miranda • José Pedro Sarmento · Carlos Augusto Mulatinho de Queiroz Pedroso

\begin{tabular}{|c|c|c|c|}
\hline Oman, Pepur, Arneric (2016). & $\begin{array}{l}\text { Questionário com } \\
\text { escala Likert de } 7 \\
\text { pontos }(n=80) \text {. }\end{array}$ & $\begin{array}{l}\text { Análise de equa- } \\
\text { ções de estrutu- } \\
\text { rais. }\end{array}$ & $\begin{array}{l}\text { Qualidade de serviço e } \\
\text { identificação. }\end{array}$ \\
\hline $\begin{array}{l}\text { Rodrigues, Sousa e Fagundes } \\
\text { (2017) }\end{array}$ & $\begin{array}{l}\text { Questionário com } \\
\text { escala Likert de } 11 \\
\text { pontos (n=352). }\end{array}$ & $\begin{array}{l}\text { Análise de equa- } \\
\text { ções estruturais. }\end{array}$ & $\begin{array}{l}\text { Identificação e satisfa- } \\
\text { ção. }\end{array}$ \\
\hline Kunkel, Funk e Lock (2017). & $\begin{array}{l}\text { Questionário com } \\
\text { escala Likert de } 7 \\
\text { pontos ( } n=414) \text {. }\end{array}$ & $\begin{array}{l}\text { Análise de equa- } \\
\text { ções estruturais. }\end{array}$ & $\begin{array}{l}\text { Associações da marca } \\
\text { da equipe e da liga. }\end{array}$ \\
\hline $\begin{array}{l}\text { Marquetto, Pinto, Grohmann e } \\
\text { Battistella (2017). }\end{array}$ & $\begin{array}{l}\text { Questionário com } \\
\text { escala Likert de } \\
5,7 \text { e } 10 \text { pontos } \\
(n=270) \text {. }\end{array}$ & $\begin{array}{l}\text { Análise de equa- } \\
\text { ções estruturais. }\end{array}$ & Amor à marca. \\
\hline Sousa e Vieira (2018) & $\begin{array}{l}\text { Questionário com } \\
\text { escala Likert de } 5 \\
\text { pontos ( } n=477) .\end{array}$ & $\begin{array}{l}\text { Correlação de } \\
\text { Spearman. }\end{array}$ & $\begin{array}{l}\text { Compromisso, con- } \\
\text { fiança, apego e satis- } \\
\text { fação. }\end{array}$ \\
\hline Silveira, Cardoso e Silva (2018) & $\begin{array}{l}\text { Questionário com } \\
\text { escala Likert de } 7 \\
\text { pontos ( } n=273) \text {. }\end{array}$ & $\begin{array}{l}\text { Análise de equa- } \\
\text { ções estruturais. }\end{array}$ & $\begin{array}{l}\text { Envolvimento e satis- } \\
\text { fação. }\end{array}$ \\
\hline Maderer e Holtbrügge (2018). & $\begin{array}{l}\text { Questionário com } \\
\text { escala Likert de } 7 \\
\text { pontos (n=3857). }\end{array}$ & $\begin{array}{l}\text { Regressão múlti- } \\
\text { pla. }\end{array}$ & $\begin{array}{l}\text { Internacionalização da } \\
\text { marca. }\end{array}$ \\
\hline $\begin{array}{l}\text { Phonthanukitithaworn e } \\
\text { Sellitto (2018). }\end{array}$ & $\begin{array}{l}\text { Questionário com } \\
\text { escala Likert de } 7 \\
\text { pontos ( } n=849) \text {. }\end{array}$ & $\begin{array}{l}\text { Análise de equa- } \\
\text { ções estruturais. }\end{array}$ & $\begin{array}{l}\text { Estética, custo, desem- } \\
\text { penho dos jogadores } \\
\text { e satisfação. }\end{array}$ \\
\hline Kościołek (2019) & $\begin{array}{l}\text { Questionário com } \\
\text { escala Likert de } 5 \\
\text { pontos ( } n=489) .\end{array}$ & $\begin{array}{l}\text { Regressão múlti- } \\
\text { pla. }\end{array}$ & Identificação. \\
\hline $\begin{array}{l}\text { Woratschek, Horbel e Popp } \\
\text { (2019) }\end{array}$ & $\begin{array}{l}\text { Questionário com } \\
\text { escala Likert de } 7 \\
\text { pontos }(n=1295) \text {. }\end{array}$ & $\begin{array}{l}\text { Análise de equa- } \\
\text { ções estruturais. }\end{array}$ & $\begin{array}{l}\text { Identificação e satisfa- } \\
\text { ção. }\end{array}$ \\
\hline $\begin{array}{l}\text { Foroughi, Ramayah e } \\
\text { Iranmanesh (2019). }\end{array}$ & $\begin{array}{l}\text { Questionário com } \\
\text { escala Likert de } \\
7 \text { e de } 5 \text { pontos } \\
(n=342) \text {. }\end{array}$ & $\begin{array}{l}\text { Análise de equa- } \\
\text { ções estruturais. }\end{array}$ & Emoções. \\
\hline
\end{tabular}

Fonte: os autores.

\section{Discussão}

Conforme dito anteriormente, o presente estudo teve como objetivo identificar quais os antecedentes da lealdade dos torcedores de clubes de futebol à luz da literatura. A ideia inicial desta discussão é tratar sobre o âmbito geral das buscas, contextos, instrumentos e análises. Posteriormente, tratar sobre os anteceden- 
A lealdade dos espectadores de futebol e seus antecedentes: uma revisão sistemática Victor Henrique Rodrigues Silva $•$ Marcos Antonio Barros Filho - Yves de Holanda Batista de Miranda • José Pedro Sarmento · Carlos Augusto Mulatinho de Queiroz Pedroso

tes encontrados como resultados dos estudos, conceituando-os com informações relevantes, sendo a ordem de apresentação de acordo com a quantidade de vezes que aparecem nos achados dos artigos selecionados.

Ao realizar a revisão sistemática, 21 artigos foram selecionados a partir dos critérios pré-definidos. A ordem cronológica nos apresenta uma boa quantidade de publicações relevantes e constantes a partir de 2012. O aumento da quantidade de estudos em um curto período pode ser explicado pela exigência dos consumidores perante as organizações de oferecerem produtos diferenciados diante de uma variedade enorme de atividades de entretenimento (SARMENTO et al., 2011).

Os estudos selecionados investigam diferentes contextos. Considera-se como um ponto positivo, pois a cultura dos indivíduos de determinado contexto pode interferir na estrutura metodológica do estudo, assim como indicar diferentes antecedentes, e devem ser considerados principalmente ao analisar o ambiente esportivo em razão do forte apego emocional (JONES et al., 2005; THEODORAKIS; ALEXANDRIS, 2008).

Ao levar em conta os critérios de seleção, não foram encontrados artigos que investigassem, de forma exclusiva, o contexto da América do Norte. Resultado que difere do estudo de Barros Filho et al. (2018), em que os EUA foram o principal contexto dos artigos selecionados. Os achados acima podem ser explicados pelo fato de que, neste estudo, buscou-se artigos que tratassem apenas de uma modalidade específica (futebol) que ainda está em desenvolvimento quando comparado com as principais modalidades do país (futebol americano, beisebol, basquetebol e hóquei no gelo).

Todos os estudos utilizaram questionários com escalas do tipo Likert. O predominante uso da escala Likert pode ser explicado por medir atitudes/comportamentos da amostra a partir do seu nível de concordância ou discordância sobre determinado item (CUNHA, 2007). Ao tratar sobre as análises, a AEE foi o método de análise estatística mais utilizado entre os estudos (17), e segundo 
A lealdade dos espectadores de futebol e seus antecedentes: uma revisão sistemática Victor Henrique Rodrigues Silva $•$ Marcos Antonio Barros Filho - Yves de Holanda Batista de Miranda • José Pedro Sarmento · Carlos Augusto Mulatinho de Queiroz Pedroso

Marôco (2014), ela é definida como uma técnica de modelagem generalizada, utilizada com o objetivo de testar a validade de modelos teóricos que buscam indicar relações causais e hipotéticas entre variáveis. Um dos grandes diferenciais desse método é conseguir operacionalizar variáveis ou construtos latentes, ou seja, que não são diretamente mensuráveis (MARÔCO, 2014). Outros três estudos utilizaram a análise de regressão, que é utilizada para analisar quais variáveis independentes predizem as variáveis dependentes (MARÔCO, 2014). Por último, apenas um utilizou a correlação de Spearman que, como o seu nome diz, é utilizada para verificar se duas variáveis se correlacionam de forma significativa do ponto de vista estatístico (BAUER, 2007).

Ao analisar os antecedentes, satisfação e identificação (inclui comunidade de torcedores) foram os que apareceram com mais frequência nos estudos sendo que, em alguns artigos, essas duas variáveis tiveram um efeito moderador que resultou em intenções comportamentais positivas (BISCAIA et al., 2012; THEODORAKIS; WANN; WEAVER, 2012; THEODORAKIS, et al., 2013; BISCAIA et al., 2013; FERNANDES; NEVES, 2014; YOSHIDA; HEERE; GORDON, 2015; RODRIGUES; SOUSA; FAGUNDES, 2017; SOUSA; VIEIRA, 2018; SILVEIRA; CARDOSO; SILVA, 2018; PHONTHANUKITITHAWORN; SELLITTO, 2018; KOŚCIOŁEK, 2019; WORATSCHEK; HORBEL; POPP, 2019).

A satisfação é a resposta prazerosa de uma série de experiências vivenciadas e é fundamental para que o torcedor tenha boas respostas em relação às situações subjetivas, e que mantenha um comportamento positivo de recompra dos serviços do clube (GREENWELL; FINK; PASTORE, 2002; MARTIN et al., 2008; YOSHIDA; JAMES, 2010). A satisfação como um dos principais antecedentes (seja como efeito moderador ou direto) corrobora uma revisão realizada por Biscaia (2016) também no contexto do futebol. Além da satisfação, a qualidade de serviço, emoções e associação à marca foram constatados em seu estudo e são encontrados neste em menor frequência. 
A lealdade dos espectadores de futebol e seus antecedentes: uma revisão sistemática Victor Henrique Rodrigues Silva $•$ Marcos Antonio Barros Filho - Yves de Holanda Batista de Miranda • José Pedro Sarmento · Carlos Augusto Mulatinho de Queiroz Pedroso

Identificação é o segundo preditor mais frequente que, no estudo de Kościołek (2019), foi o antecedente mais forte entre os torcedores de clubes de futebol da liga polonesa. Ter a sua identidade relacionada a um clube ou a uma comunidade de torcedores pertencentes ao clube é um fator primordial para um comportamento positivo e forte lealdade, pelo fato de o indivíduo se envolver nas atividades diárias do clube e sempre buscar dar o seu máximo para que o grupo ou clube ao qual ele pertence eleve o seu status (MCMILLAN; CHAVIS, 1986; WORATSCHEK; HORBEL; POPP, 2019). A criação de uma identidade esportiva pode ser considerada a internalização de uma equipe esportiva no self-concept do indivíduo, intensificada de acordo com o nível que um objeto esportivo satisfaz suas necessidades (i.e., pertencimento a um grupo, autoestima, melhora da imagem pública etc.) (FUNK; JAMES, 2004).

Outros antecedentes também foram citados nos diferentes estudos selecionados: associações da marca (inclusas no valor da marca e amor à marca), internacionalização da marca, emoções (inclui alegria), qualidade de serviço (inclui custo/preço, desempenho dos jogadores, segurança, relacionamento e estética), confiança, envolvimento e compromisso.

A associação da marca é definida como o significado de uma marca na mente do consumidor (KELLER, 1993). Diferentes associações podem ser criadas na mente do consumidor, por isso sua análise pode ocorrer a partir de diferentes dimensões, como enfatizado por Ross, James e Vargas (2006), Gladden e Funk (2002) e Kunkel, Funk e King (2014). As associações são importantes para o consumidor criar a imagem da marca em sua mente, o que pode influenciar no seu comportamento (racional, emocional, entre outros) (RODRIGUES, 2010). Além disso, também exercem ligação para o desenvolvimento de atitudes favoráveis, que promove a lealdade do indivíduo em questão (FUNK; JAMES, 2006; KUNKEL; FUNK \& HILL, 2013).

A literatura não tem um conceito concreto para as emoções por causa da sua subjetividade, mas sabe-se que é um componente que precisa ser considerado no esporte pelo papel desem- 
A lealdade dos espectadores de futebol e seus antecedentes: uma revisão sistemática Victor Henrique Rodrigues Silva • Marcos Antonio Barros Filho • Yves de Holanda Batista de Miranda • José Pedro Sarmento · Carlos Augusto Mulatinho de Queiroz Pedroso

penhado (DIAS, 2005; BISCAIA et al., 2014). Oatley e Jenkins (2000) definem emoções como uma resposta que engloba três fatores: cognitivo, fisiológico e emocional. Sendo a participação em um evento esportivo estimuladora de diferentes respostas emocionais nos indivíduos, elas são capazes de causar impactos positivos e negativos em relação ao seu comportamento (HOLT, 1995).

Qualidade de serviço é uma dimensão ampla, com itens que variam de acordo com o objetivo de cada estudo. É a resposta positiva da percepção global das ações que a entidade presta ao seu público-alvo (BISCAIA, 2016; BISCAIA et al., 2014). Essas ações que a organização realiza podem ser chamadas de serviços auxiliares ou serviços principais. Os serviços auxiliares são as atividades periféricas ao serviço principal (segurança, estética, relacionamento, preço, entre outros) e o serviço principal é entendido como o jogo propriamente dito (desempenho dos jogadores), com grande capacidade de alterar a tal percepção global do serviço (YOSHIDA; JAMES, 2010).

Analisada e definida como antecedente em dois dos estudos selecionados (SOUZA; VIEIRA, 2018; EBRAHIMI; FADAEI, 2016), a confiança assume um papel de elevada importância no processo de lealdade pelo fato de ser considerada uma troca entre o consumidor e a organização que leva o torcedor a consumir com o sentimento de que o serviço será entregue da forma que ele espera e, como consequência, os níveis de comportamentos favoráveis elevam (MORGAN; HUNT, 1994; HARRIS; OGNOBBA, 2008).

No mesmo estudo de Ebrahimi e Fadei (2016), além do de Silveira, Cardoso e Silva (2018), o envolvimento é uma variável preditora relevante para compreender as intenções comportamentais favoráveis perante uma determinada prática ou organização esportiva, e é entendido como um fator motivacional que leva o indivíduo a participar das atividades do clube com maior interesse, principalmente no âmbito esportivo, pelo fato de que nenhum cliente tem o apego emocional tão forte quanto os torcedores (FUNK; RIDINGER; MOORMAN, 2004). 
A lealdade dos espectadores de futebol e seus antecedentes: uma revisão sistemática Victor Henrique Rodrigues Silva $•$ Marcos Antonio Barros Filho - Yves de Holanda Batista de Miranda • José Pedro Sarmento · Carlos Augusto Mulatinho de Queiroz Pedroso

Por último, o compromisso também foi identificado como preditor para as intenções comportamentais favoráveis em um estudo (SOUZA; VIEIRA, 2018). Morgan e Hunt (1994) já destacavam essa variável no marketing relacional entre organização e cliente. Compromisso define-se como o processo de troca entre as promessas da organização cumpridas e as expectativas atingidas ou superadas por parte do cliente que, como consequência, aumentará os seus níveis de intenções comportamentais (BERRY, 1995). Isso gera o desejo de manter uma relação de ambas as partes por tempo indeterminado (MORGAN; HUNT, 1994).

É de suma importância que os gestores de clubes e organizações esportivas conheçam o papel das dimensões que influenciam positivamente na lealdade do torcedor. Quanto mais essas dimensões são estimuladas por ações positivas e planejadas por parte da organização, mais chances o indivíduo tem de se tornar leal, independente das possíveis alterações no ambiente externo e interno.

\section{Conclusões}

Conclui-se que a satisfação e identificação foram os antecedentes apresentados com maior frequência nos estudos, tanto como influente no comportamento leal de forma direta quanto de forma indireta (efeito mediador). Vale ressaltar que outros preditores, como associações da marca, emoções, qualidade de serviço, confiança, envolvimento e compromisso, também foram apresentados com resultados significativos em alguns estudos selecionados.

Os resultados deste estudo contribuem para a área ao apresentar os principais antecedentes aos gestores de organizações esportivas para que sejam diretamente estimulados em seu público-alvo pelo fato de que o comportamento leal do consumidor é fundamental no organismo de qualquer organização. No campo acadêmico, a metodologia aplicada contribui para a elaboração de 
A lealdade dos espectadores de futebol e seus antecedentes: uma revisão sistemática Victor Henrique Rodrigues Silva $•$ Marcos Antonio Barros Filho - Yves de Holanda Batista de Miranda • José Pedro Sarmento · Carlos Augusto Mulatinho de Queiroz Pedroso

estudos sobre lealdade em outros contextos, além de estimular novas publicações de caráter original. Como sugestões, a elaboração de estudos que analisem os antecedentes da lealdade dos torcedores de clubes de futebol nos contextos das Américas do Sul e do Norte, em especial no Brasil, e recomenda-se que sejam realizadas pesquisas nas mais diversas modalidades esportivas. Além disso, sugere-se a realização dos estudos longitudinais para o fortalecimento da temática.

\section{Referências bibliográficas}

BARROS FILHO, M. A. et al. Qualidade de serviços e satisfação de espectadores de eventos esportivos: uma revisão sistemática.

Movimento (ESEFID/UFRGS), p. 1381-1394, 2018.

BATISTA, P.; CUNHA, M. Investigação qualitativa em desporto. Porto: Centro de Investigação, Formação e Inovação e Intervenção em Desporto, 2013.

BAUER, H. H.; SAUER, N. E.; SCHMITT, P. Customer-based brand equity in the team sport industry. European Journal of Marketing, p. 496-513, 2005.

BAUER, L. Estimação do Coeficiente de Correlação de Spearman Ponderado. Dissertação apresentada para obtenção do grau de Mestre em Epidemiologia. Faculdade de Medicina da Universidade Federal do Rio Grande do Sul, 2007.

BERRY, L. L. Relationship Marketing of Services: Growing interest, emerging perspectives. Journal of The Academy of Marketing Science, p. 236-245, 1995.

BISCAIA, R. Revisiting the role of football spectator's behavioral intentions and its antecedents. The Open Sports Science Journal, p. 3-12, 2016.

BISCAIA, R. Revisiting the role of football spectators' behavioral intentions and its antecedentss. The Open Sports Sciences Journal, p. 3-12, 2016. 
A lealdade dos espectadores de futebol e seus antecedentes: uma revisão sistemática Victor Henrique Rodrigues Silva $•$ Marcos Antonio Barros Filho - Yves de Holanda Batista de Miranda • José Pedro Sarmento · Carlos Augusto Mulatinho de Queiroz Pedroso

BISCAIA, R. et al. The effects of emotions on football spectator's satisfaction and behavioural intentions. European Sport Management Quarterly, p. 227-242, 2012.

BISCAIA, R. et al. The role of service quality and ticket pricing on satisfaction and behavioral intention within professional football. Journal of Sport Marketing \& Sporsonship, p. 301-326, 2013. BISCAIA, R. et al. Estudo da Lealdade dos Espectadores. Recife: UFPE, 2014.

BISCAIA, R. et al. Investigating the role of fan club membership on perceptions of team brand equity in football. Sport Management Review, 2015.

CHELLADURAI, A.; CHANG, K. Targets and standards of quality in sport services. Sport Management Review, p. 1-22, 2000.

CUNHA, L. M. A. Modelos Rasch e Escalas de Likert e Thurstone na medição de atitudes. Porto: Universidade de Lisboa, 2007.

DIAS, C. Do stress e ansiedade às emoções no desporto. Da importância da sua compreensão à necessidade da sua gestão. Dissertação apresentada para obtenção do grau de Doutor em Psicologia. Instituto de Educação e Psicologia da Universidade do Minho., 2005.

EBRAHIMI, P.; FADAEI, M. The Impact of Relationship Marketing on Team Loyalty (The Case Study: Sport Team Fans of Azadeghan Football League of Iran). International Journal of Medical Research \& Health Sciences, p. 52-68, 2016.

FERNANDES, T.; NEVES, S. The role of serviscape as a driver of customer value in experience-centric service organizations: the Dragon Football Stadium case. Journal of Strategic Marketing, p. 548-560, 2014.

FOROUGHI, B. et al. Impact of core product quality on sport fan's emotions and behavioral intentions. International Journal of Sports Marketing and Sporsonship, p. 110-129, 2016.

FOROUGHI, B.; RAMAYAH, K.; IRANMANESH, M. The effects of pheripheral service quality on spectator's emotions and behav- 
A lealdade dos espectadores de futebol e seus antecedentes: uma revisão sistemática Victor Henrique Rodrigues Silva $•$ Marcos Antonio Barros Filho - Yves de Holanda Batista de Miranda • José Pedro Sarmento · Carlos Augusto Mulatinho de Queiroz Pedroso

ioral intentions. International Journal of Sports Marketing and Sporsonship, 2019.

FUNK, D. C.; JAMES , J. D. The Fan Attitude Network (FAN) Model: Exploring attitude formation and change among sport consumers.. Sport Management Review, p. 1-26, 2004.

FUNK, D. C.; PASTORE, D. L. Equating attitudes to allegiance: The usefulness of selected attitudinal information in segmeting loyalty to professional sports teams. Sport Marketing Quarterly, p. 175-184, 2000.

FUNK, D.; JAMES, J. D. Consumer loyalty: The meaning of attachment in the development of sport team allegiance. Journal of Sport Management, p. 189-217, 2006.

FUNK, D.; RIDINGER, L.; MOORMAN, A. The psychological continuum model: a conceptual framework for understanding an individual's psychological connection to Sport. Sport Management Review, p. 119-150, 2004.

GOMES, I.; CAMINHA, I. Guia para estudos de revisão sistemática: Uma opção metodológica para as ciências do movimento humano. Movimento (ESEFID/UFRGS), p. 395-411, 2013.

GREENWELL, T.; FINK, J.; PASTORE, D. Assessing the influence of the physical sports facility on customer satisfaction within the context of the service experience. Sport Management Review, p. 129-148, 2002.

HARRIS, L. C.; OGBONNA, E. The dynamics underlyng service firm-customer relationships: insights from a study of English Premier League soccer fans. Journal of Service Research , p. 382-399, 2008.

HOLT, D. How consumers consume: a typology of consumption pratices. Journal of Consumer Research, p. 1-16, 1995.

JONES, M. et al. Development and validation of the Sport Emotion Questionnaire. Journal of Sport \& Exercise Psycology, p. 407431, 2005. 
A lealdade dos espectadores de futebol e seus antecedentes: uma revisão sistemática Victor Henrique Rodrigues Silva $•$ Marcos Antonio Barros Filho - Yves de Holanda Batista de Miranda • José Pedro Sarmento · Carlos Augusto Mulatinho de Queiroz Pedroso

KELLER, K. L. Conceptualizing, Measuring, and Managing Customer-Based Brand Equity. Journal of Marketing, p. 1-22, 1993.

KOśCIOłEK, S. Consumer loyalty among fans of sports clubs: How much do they vary across disciplines? Baltic Journal of Health and Physical Activity, p. 67-77, 2019.

KUNKEL, T.; FUNK, D.; HILL, B. Brand Architecture, Drivers of Consumer Involvement, and Brand Loyalty With Professional Sport Leagues and Teams. Journal of Sport Management, $p$. 177-192, 2013.

KUNKEL, T.; FUNK, D.; LOCK, D. The Effect of League Brand on the Relationship Between the Team Brand and Behavioral Intentions: A formative Approach Examining Brand Assosioations and Brand Relationships. Journal of Sport Management, p. 317-332, 2017.

MADERER, D.; HOLTBRÜGGE, D. International activies of football clubs, fan attitudes, and brand loyalty. Journal of Brand Management, 2018.

MARÔCO, J. Análise de equações estruturais: Fundamentos teóricos, softwares \& aplicações. $2^{a}$ ed. Pêro Pinheiro: ReportNumber, 2014a.

MARQUETTO, M. et al. Knowing the Fans Behaviour in Relation To Love of Football Clubs Brands. Brazilian Business Review, p. 272-287, 2017.

MARTIN, D. et al. The role of emotions in explaining consumer satisfaction and future behavioral intention. Journal of Services Marketing, p. 224-236, 2008.

MCMILAN, D.; CHAVIS, D. Sense of community: A definition and theory. Journal of Community Psychology, p. 6-23, 1986.

MITTAL, V.; KUMAR, P.; TSIROS, M. Atribute-Level Performance, Satisfaction, and Behavioral Intentions over time: A ConsumptionSystem Approach. Journal of Marketing, p. 88-101, 1999.

MORGAN, R. M.; HUNT , S. D. The commitment trust theory of relationship marketing. Journal of Marketing, p. 20-38, 1994. 
A lealdade dos espectadores de futebol e seus antecedentes: uma revisão sistemática Victor Henrique Rodrigues Silva $•$ Marcos Antonio Barros Filho - Yves de Holanda Batista de Miranda • José Pedro Sarmento · Carlos Augusto Mulatinho de Queiroz Pedroso

OATLEY, K.; JENKINS, J. Understanding Emotions. Cambridge: Blackwell Scientific, 1996.

OMAN, B.; PEPUR, M.; ARNERIC, J. The Impact of Service Quality and Sport-Team Idenfication on the Repurchase Intention.

Management, p. 19-46, 2016.

PHONTHANUKITITHAWORN, C.; SELLITTO, C. Perceptions of service quality at football stadiums: influence on fan's intentions to attend future games. Managing Sport and Leisure, p. 204-224, 2018.

RODRIGUES , P. C. Importância das associações à marca na construção do capital da marca. Economia \& Empresa, p. 205-231, 2010.

RODRIGUES, R.; SOUSA, C.; FAGUNDES, A. Aspectos emocionais e experienciais influenciadores da ida do torcedor aos estádios de futebol de Belo Horizonte-MG. Revista Brasileira de Marketing, p. 31-48, 2018.

SARMENTO, J. P. et al. O evento desportivo como factor de desenvolvimento. Revista Intercontinental de Gestão Desportiva, p. 1-14, 2011.

SILVEIRA, M.; CARDOSO, M.; SILVA, F. Factors influencing attendance at stadiums and arenas. Marketing Intelligence \& Planning, p. 1-16, 2018.

SOUZA, B.; VIEIRA, C. Um estudo sobre a afetividade aplicada ao fenómeno do futebol: Abordagem ótica dos consumidores. PODIUM Sport, Leisure and Tourism Review, p. 293-312, 2018. THEODORAKIS, N. et al. Predicting spectator's behavioural intentions in professional football: The role of satisfaction and service quality. Sport Management Review, p. 85-96, 2013.

THEODORAKIS, N.; ALEXANDRIS, K. Can service quality predct spectarios behavioral intentions in professional soccer?

Managing Leidre, 2008.

THEODORAKIS, N.; WANN, D.; WEAVER, S. An Antecedent Model of Team Identification in the Context of Professinal Soccer. Sport Marketing Quartertly, p. 80-90, 2012. 
A lealdade dos espectadores de futebol e seus antecedentes: uma revisão sistemática Victor Henrique Rodrigues Silva $•$ Marcos Antonio Barros Filho - Yves de Holanda Batista de Miranda • José Pedro Sarmento · Carlos Augusto Mulatinho de Queiroz Pedroso

THEYSOHN, S. et al. Official supporters clubs: the untapped potential of fan loyalty. International Journal of Sport Marketing \& Sporsonship, p. 302-324, 2009.

WORASTCHECK, H.; HORBEL, C.; POPP, B. Determining customer satisfaction and loyalty from a value co-creation perspective. The Service Industrie Journal, p. 1-24, 2019.

YOSHIDA, M.; GORDON, B. Who is more influenced by customer equity drivers? A moderator analysis in a professional soccer context. Sport Management Review, p. 389-403, 2012.

YOSHIDA, M.; HERE, B.; GORDON, B. Predicting Behavioral Loyalty Through Community: Why Other Fans Are More Important Than Our Own Intentions, Our Satisfaction, and the Team Itself. Journal of Sport Management, p. 318-333, 2015.

YOSHIDA, M.; JAMES , J. D. Service quality at sporting events: Is a esthetic quality a missing dimension? Sport Management Review, p. 13-24, 2011.

YOSHIDA, M.; JAMES, J. D. Customer Satisfaction With Game and Service Experiences: Antecedents and Consequences. Journal of Sport Management, p. 338-361, 2010.

ZINS, A. Relative attitudes and commitment in custome loyalt models. Journal of Service Industry Management, p. 269-294, 2001.

\section{Publisher}

Universidade Federal de Goiás. Faculdade de Educação Física e Dança. Publicação no Portal de Periódicos UFG. As ideias expressadas neste artigo são de responsabilidade de seus autores, não representando, necessariamente, a opinião dos editores ou da universidade. 